\title{
Comparative QTL analysis for yield components and morphological traits in maize (Zea mays L.) under water-stressed and well-watered conditions
}

\author{
Xiaoqiang Zhao ${ }^{1,2)}$, Jinwen Zhang, ${ }^{1,2)}$, Peng Fang ${ }^{1,2)}$ and Yunling Peng*1,2) \\ 1) Gansu Provincial Key Laboratory of Aridland Crop Science, Gansu Agricultural University, Lanzhou 730070, China \\ 2) College of Agronomy, Gansu Agricultural University, Lanzhou 730070, China
}

\begin{abstract}
Drought significantly influences maize morphology and yield potential. The elucidation of the genetic mechanisms of yield components and morphological traits, and tightly linked molecular markers under drought stress are thus of great importance in marker assisted selection (MAS) breeding. Here, we identified 32 QTLs for grain weight per ear, kernel ratio, and ear height-to-plant height ratio across two $\mathrm{F}_{2: 3}$ populations under both drought and non-drought conditions by single-environment mapping with composite interval mapping (CIM), of which 21 QTLs were mapped under water-stressed conditions. We identified 29 QTLs by joint analysis of all environments with mixed-linear-model-based composite interval mapping (MCIM), 14 QTLs involved in QTL-by-environment interactions, and 11 epistatic interactions. Further analysis simultaneously identified 20 stable QTLs (sQTLs) by CIM and MCIM could be useful for genetic improvement of these traits via QTL pyramiding. Remarkably, bin 1.07-1.10/6.05/8.03/8.06 exhibited four pleiotropic sQTLs that were consistent with phenotypic correlations among traits, supporting the pleiotropy of QTLs and playing important roles in conferring growth and yield advantages under contrasting watering conditions. These findings provide information on the genetic mechanisms responsible for yield components and morphological traits that are affected by different watering conditions. Furthermore, these alleles provide useful targets for MAS.
\end{abstract}

Key Words: maize, QTL, yield components, morphological traits, drought.

\section{Introduction}

Maize (Zea mays L.) is one of the most important crops worldwide that is utilized as food, animal feed, and raw material of bioenergy. Drought is considered one of the most important abiotic stresses that influence global maize production. Drought stress can adversely affect various aspects of maize physiological metabolism and growth, including photosynthesis, plant growth, tassel development, dry matter production, and grain yield formation (Ge et al. 2012), even causing a $39.3 \%$ reduction in maize yield from 1980 to 2015 using global estimates of drought effects (Daryanto et al. 2016). Maize undergoes various morphological, biochemical and physiological changes to respond and adapt to drought stress (Almeida et al. 2014). Moreover, the genetic improvement for drought tolerance can ensure sustainable and long-term benefits (Duvick 2005). Therefore, drought-tolerant maize varieties are urgently needed to

Communicated by Hiroyoshi Iwata

Received February 26, 2018. Accepted August 1, 2019.

First Published Online in J-STAGE on October 31, 2019.

*Corresponding author (e-mail: pengyunlingpyl@163.com) maintain sufficient global food production.

Maize grain yield (GY) is the primary objective of breeding for drought tolerance, however, the low heritability of GY, as it decreases under drought stress conditions, makes the direct selection for this trait inefficient (Su et al. 2017). However, multiple morphological traits and yield components, namely, plant height $(\mathrm{PH})$, ear height $(\mathrm{EH})$, leaf area (LA), anthesis-silking interval (ASI), tassel branch number (TBN), ear weight (EW), cob weight (CW), 100-kernel weight (KW), and ear length (EL), are correlated with GY and drought tolerance can experience increased genetic variance and heritability under drought environments (Almeida et al. 2014, Zhao et al. 2017, 2018b, Ziyomo and Bernardo 2012). Moreover, drought stress inhibits growth of vegetative organs, limits photosynthesis, reduces flux of assimilates to the developing ears, slows down ear and silk growth and delays silk emergence (Bänziger et al. 2000, Messmer et al. 2009, Zhao et al. 2017). Therefore, these traits may be more suitable for identifying and improving maize selection responses to drought.

Genetic studies on maize PH, EH, ASI, TBN, EW, CW, $\mathrm{KW}$, and EL have been extensively conducted using quantitative trait locus (QTL) mapping in biparental populations 
during drought (Almeida et al. 2014, Lu et al. 2010, Messmer et al. 2009, Peng et al. 2011, Semagn et al. 2013, Tan et al. 2011, Vargas et al. 2006, Zhao et al. 2017). QTLs/ genes have been identified for morphological traits and yield components in maize using map-based cloning strategies, such as Brachytic 2 (Br2) (Xing et al. 2015), gln 1-4 (Martin et al. 2006), Opaque-2 (o2) (Schmidt et al. 1992), unbranched2 (ub2) (Chuck et al. 2014), and ZmCLA4 (Zhang et al. 2014), which can be useful targets for marker assisted selection (MAS) in modern maize breeding programs. In addition, there is growing evidence suggesting that ear height-to-plant height ratio (EHPH), grain weight per ear $(\mathrm{GW})$, and kernel ratio (KR) are closely associated with drought tolerance and yield, EHPH (phenotypic correlation coefficient $\left.\left(r_{p}\right)=0.318 \sim 0.578\right)$, GW $\left(r_{p}=0.979 \sim\right.$ $0.999)$, and KR ( $\left.r_{p}=0.629 \sim 0.688\right)$ showed significantly positive correlation to GY, and EHPH had significantly positive correlation to $\mathrm{GW}\left(r_{p}=0.308 \sim 0.3111\right)$ and $\mathrm{KR}$ $\left(r_{p}=0.241 \sim 0.262\right)$ under both water regimens (Begna et al. 2000, Du et al. 2011, 2012, He et al. 2016, Khush 1999, Meng et al. 2016, Zhao 2018). Therefore, higher GW and KR can be achieved by maintaining a suitable EHPH under drought stress. However, a limited number of QTLs for EHPH, GW, and KR have been identified under different levels of water availability (Chang et al. 2017, Dong et al. 2015, Ku et al. 2015, Zhang et al. 2013), and the genetic mechanisms underlying these three traits remains poorly understood, thus warranting in-depth investigations. Furthermore, a better understanding of the genotype-by-environment $(\mathrm{G} \times \mathrm{E})$ interaction will provide a foundation for the genetic improvement and optimization of genotypes across different environments (EI-Soda et al. 2014).

The overall objective of the present study was to identify QTLs responsible for EHPH, $\mathrm{GW}$, and $\mathrm{KR}$ in two maize $\mathrm{F}_{2: 3}$ populations subjected to both water regimens at Wuwei in 2014, Zhangye in 2014, Gulang in 2015, and Jingtai in 2015 by single-environment mapping with composite interval mapping (CIM), respectively. Then, joint QTLs, epistatic interactions, and QTL-by-environment $(\mathrm{QTL} \times \mathrm{E})$ interactions were identified across all environments using mixedlinear-model based composite interval mapping (MCIM). By combining these experiments, we hope to identify hot spots and areas of co-localization across experiments, which may further contribute to mapping QTLs, revealing genetic mechanisms for EHPH, GW, and KR in drought-stressed and unstressed conditions, and developing MAS breeding for EHPH, GW, and KR to improve drought tolerance and yield in maize.

\section{Materials and Methods}

\section{Plant materials}

The two sets of study populations comprised 202 (POP1) and 218 (POP2) $\mathrm{F}_{2: 3}$ families, which were derived from the Langhuang $\times$ TS141 (H1) and Chang7-2 $\times$ TS141 (H2) crosses, respectively, and were used to identify QTLs for
EHPH, GW, and KR under both water-stressed and wellwatered conditions, respectively. The parents of the two $\mathrm{F}_{2: 3}$ populations were selected based on their distinct maize germplasm groups and drought tolerance. The drought-tolerant inbred lines Langhuang and Chang7-2 originated from the Tangsipingtou (TSPT) germplasm and used as female parents, and the drought-sensitive inbred line TS141 was derived from the Reid yellow dent (Reid) germplasm and used as the common male parent (Peng et al. 2014, Zhao et al. 2017, 2018a, 2018b).

\section{Field trials and trait evaluation}

The two parents (Langhuang and TS141), corresponding to the $\mathrm{F}_{1}$ hybrid (H1) and $202 \mathrm{~F}_{2: 3}$ families (POP1), were planted at two sites in Gansu Province, China, namely Wuwei $\left(37.97^{\circ} \mathrm{N}, 102.63^{\circ} \mathrm{E} ; 1,508 \mathrm{~m}\right.$ altitude; sand-loam) and Zhangye $\left(38.83^{\circ} \mathrm{N}, 106.93^{\circ} \mathrm{E}\right.$; $1,536 \mathrm{~m}$ altitude; sandloam) in 2014. The other two parents (Chang7-2 and TS141), corresponding to the $F_{1}$ hybrid (H2) and $218 F_{2: 3}$ families (POP2), were evaluated at two other sites in Gansu Province, China, namely Gulang $\left(37.67^{\circ} \mathrm{N}, 102.85^{\circ} \mathrm{E}\right.$; $1,785 \mathrm{~m}$ altitude; sand-loam) and Jingtai $\left(37.18^{\circ} \mathrm{N}, 104.03^{\circ}\right.$ E; 1,640 m altitude; sand-loam) in 2015. We recorded the weather data at Wuwei in 2014, Zhangye in 2014, Gulang in 2015, and Jingtai in 2015, respectively (Supplemental Fig. 1). Well-watered (WW, WZ, WG, and WJ) and waterstressed (SW, SZ, SG, and SJ) experiments were conducted at Wuwei, Zhangye, Gulang, and Jingtai following a randomized block design that included three replicates. Plastic film $(0.08 \mathrm{~mm}$ thick and $120 \mathrm{~cm}$ wide $)$ was manually laid out to cover the soil at the four sites. The plants were managed by drip irrigation. In each plot, a single $F_{2: 3}$ family, parent, or $F_{1}$ hybrid was planted in a row that was $6 \mathrm{~m}$ long and $0.6 \mathrm{~m}$ wide, with a total of 20 plants at a density of 55,580 plants ha ${ }^{-1}$. The Langhuang, Chang7-2, TS141, H1, H2, POP1, and POP2 plants under four well-watered conditions (WW, WZ, WG, and WJ) were irrigated when rainfall was insufficient during all developmental stages. Waterstressed Langhuang, Chang7-2, TS141, H1, H2, POP1, and POP2 plants under four water-stressed conditions (SW, SZ, $\mathrm{SG}$, and SJ) were not irrigated from the beginning of the expected V18 stage to the end of the expected R1 stage, although these plants were irrigated at 20-d intervals at other developmental stages. The V18 stage is where the silks from the basal ear ovules elongate first, and brace roots (aerial nodal roots) grow from the nodes above the soil surface, whereas at the R1 stage, silks are visible outside the husks (Ritchie et al. 1997). Insufficient moisture from the V18 to R1 stages can cause poor pollination and kernel set.

For the Langhuang, Chang7-2, TS141, H1, H2, POP1, and POP2 plants under each watering condition, ASI (number of days between $50 \%$ pollen shedding to $50 \%$ silking, d) was assessed in each plot. Five days after pollen shedding, 10 consecutive plants were selected from the middle of each plot to evaluate PH (height from the ground surface to the tassel tip, $\mathrm{cm}$ ) and $\mathrm{EH}$ (height from the ground surface to 
the node bearing the highest ear, $\mathrm{cm}$ ), which were then used in the calculation for EHPH with the following equation (Dong et al. 2015):

$$
\mathrm{EHPH}=\mathrm{EH} / \mathrm{PH}
$$

We then harvested the corresponding plants from each plot, which were subsequently air-dried to determine EW (weight per ear including the cob, g), GW (grain weight per ear on a scale, g), KW (100 kernels randomly selected from the bulked kernels, g), CW (weight per cob on a fine scale, g), EL (length per ear including the bald head, cm). These values were then used in calculating for KR using the following equation (Chang et al. 2017):

$$
\mathrm{KR}=\mathrm{GW} / \mathrm{EW} \times 100 \%
$$

Relative to the well-watered conditions, we also calculated the average rate of change (RC) of each trait under waterstressed conditions using the following equation (Zhao et al. 2018b):

$$
\mathrm{RC}=\left(1-T_{S} / T_{W}\right) \times 100 \%
$$

where $T_{S}$ is the average value of a trait under water-stressed conditions, and $T_{W}$ is the average value of the corresponding trait under well-watered conditions.

\section{Phenotypic data analysis}

The collected phenotypic data from each watering condition were statistically analyzed. The general linear model for univariate (GLM-Univariate) tests was used for conducting an ANOVA (Zhao et al. 2017), which was employed to evaluate the total and residual variances among $\mathrm{F}_{2: 3}$ populations for each trait. Pearson correlation analyses and dendrogram construction were performed using the IBM-SPSS Statistics 19.0 software (SPSS Inc., Chicago, IL, USA). Broad-sense heritability $\left(H^{2}\right)$ was calculated using the following equation (Knapp et al. 1985):

$$
H^{2}=\sigma_{g}^{2} /\left(\sigma_{g}^{2}+\sigma_{g e}^{2} / n+\sigma^{2} / n r\right) \times 100 \%
$$

where $\sigma_{g}^{2}$ is the genetic variance, $\sigma_{g e}^{2}$ is the $\mathrm{G} \times \mathrm{E}$ variance, $\sigma^{2}$ is the error variance, $r(r=3)$ is the replication number, and $n(n=2)$ is the number of environments.

\section{Genetic linkage map construction and QTL analysis}

A total of 213/217 genome-wide polymorphic simple sequence repeats (SSRs) were identified between Langhuang/ Chang7-2 and TS141 using the MaizeGDB (http://www. maizegdb.org/), which were subsequently used to genotype the 202/218 $F_{2}$ individuals and establish two corresponding genetic linkage maps (Zhao et al. 2017). The two genetic linkage maps were $1,542.5$ and $1,648.8 \mathrm{cM}$ in length, with an average interval of 7.8 and $8.0 \mathrm{cM}$, respectively.

The identification of QTLs for each trait in each watering environment was performed using Windows QTL Cartographer software version 2.5 with CIM approach (Wang et al. 2007). For CIM, model 6 of the Zmapqtl module was used to identify QTLs. Window size was $10 \mathrm{cM}$, and cofactors were selected through forward and backward regressions with the in and out thresholds at a $P<0.05$. A genome-wide critical threshold value was estimated for experiment-wise type I error rate of 0.05 with 1,000 random permutations (Trachsel et al. 2009). The genetic action of each QTL was estimated by Stuber et al. (1987) as: $|\mathrm{d} / \mathrm{a}|=\mid$ dominance/additive|; A, additive $(|\mathrm{d} / \mathrm{a}|=0.00 \sim 0.20)$; $\mathrm{PD}$, partial dominance $(|\mathrm{d} / \mathrm{a}|=0.21 \sim 0.80) ; \mathrm{D}$, dominance $(|\mathrm{d} / \mathrm{a}|=0.81 \sim 1.20)$; OD, over-dominance $(|\mathrm{d} / \mathrm{a}|>1.20)$.

The joint QTL, epistatic interaction between QTLs for each trait at each watering environment and QTL $\times$ E interaction across all watering environments were identified using QTL Network version 2.0, with MCIM approach (Yang et al. 2016). The testing window, walk speed, and filtration window of the genome scan were set at 10,2 , and $10 \mathrm{cM}$, respectively. A total of 1,000 permutations were performed to determine the threshold logarithm (base 10) of odds ratio (LOD), for each trait for declaring a significant QTL at a $P<0.05$ probability level. The name of the QTL was assigned according to the modifying nomenclature of McCouch et al. (1997). Moreover, the letter 'J' was added into the middle of this QTL name (namely, inserted between trait abbreviation and chromosome number), whereas one QTL was only detected in joint analysis with MCIM but not in a single environment through the CIM program. The confidence interval (CI) of the identified QTL was estimated using the following equation (Darvasi and Soller 1997):

$$
\mathrm{CI}=530 /\left(N \times R^{2}\right)
$$

where $\mathrm{N}$ is the population size, and $R^{2}$ is the value of the phenotypic variation contributed by the QTL. QTLs for tested traits were detected within the same marker interval or within overlapping CIs. The corresponding loci were assumed to be common QTLs with pleiotropic effects. QTLs that were repeatedly detected with CIM/MCIM in different environments or different mapping populations were considered stable QTLs (sQTLs).

\section{Results}

\section{Phenotypic variations under water-stressed conditions}

We analyzed the phenotypic values of the three parents (Langhuang, Chang7-2, and TS141), two $\mathrm{F}_{1}$ hybrids (H1 and $\mathrm{H} 2$ ), and two $\mathrm{F}_{2: 3}$ populations (POP1 and POP2) under both well-watered (WW, WZ, WG, and WJ) and waterstressed (SW, SZ, SG, and SJ) conditions, respectively. Under well-watered conditions, except for KR, other nine traits of the common male parent (TS141) were larger than the female parents (Langhuang and Chang7-2) (Supplemental Table 1). This finding shows that these phenotypic traits were highly significantly different between the male 


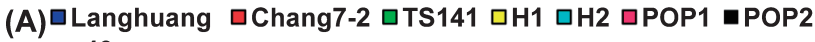

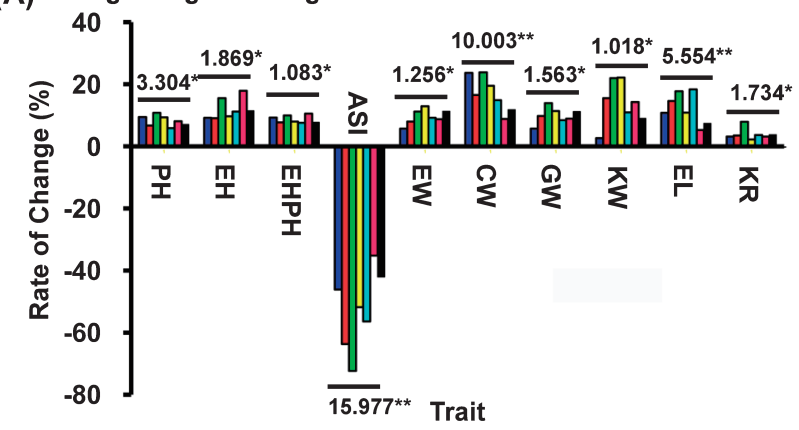

(B)

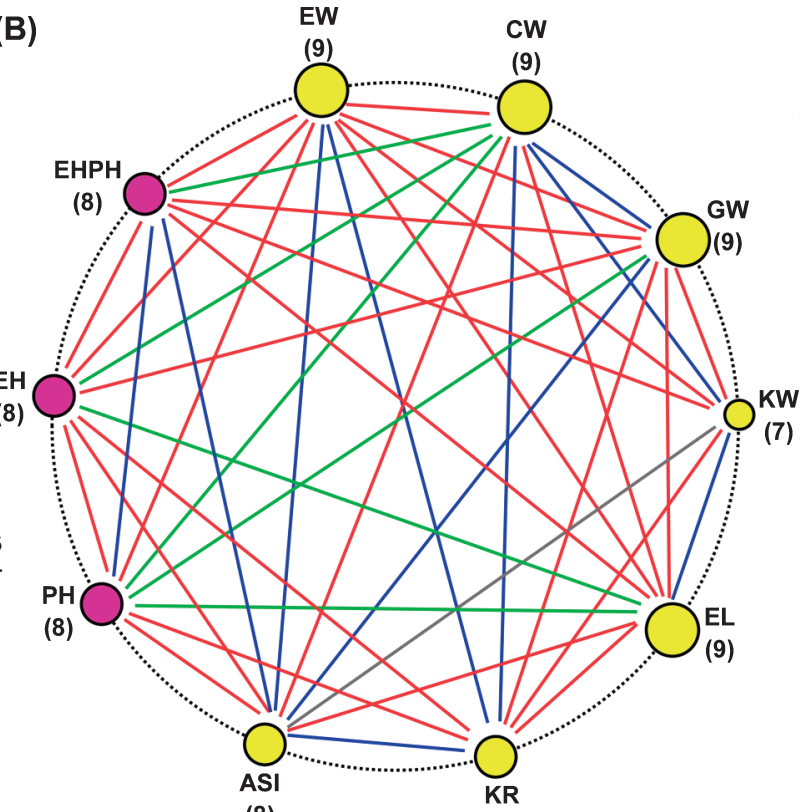

(8)

(8)

Fig. 1. Extensive phenotypic variations, correlation, and dendrogram in 10 maize traits. (A) Average rate of changes (RC) for 10 tested traits in three parents (Langhuang, Chang7-2, and TS141), two $\mathrm{F}_{1}$ hybrids (H1 and H2), and two $\mathrm{F}_{2: 3}$ populations (POP1 and POP2) under water-stressed conditions, respectively. $F$-value**/* means the significant difference at $P<0.01$ or $P<0.05$ via ANOVA. (B) Correlation among 10 maize traits. Red/blue lines designate positive/negative correlations between two traits $(P<0.01)$, and green/gray lines designate positive/negative correlations between two traits $(P<0.05)$, respectively. Circles of different sizes reflect the number of corresponding traits. (C) Dendrogram of all 10 maize traits. The scale indicates Euclidean distance, which was calculated based on the standardized phenotypic data across three parents, two $\mathrm{F}_{1}$ hybrids, and two $\mathrm{F}_{2: 3}$ populations, respectively.

and female parents. Under water-stressed conditions, $\mathrm{PH}$, $\mathrm{EH}, \mathrm{EHPH}, \mathrm{EW}, \mathrm{CW}, \mathrm{GW}, \mathrm{KW}$, and EL significantly decreased in Langhuang, Chang7-2, TS141, H1, H2, POP1, and POP2, and KR exhibited a slight decrease. ASI, however, displayed a significant increase. Further analysis indicated that the drought-sensitive male parent TS141 had more changes (RC) in all 10 tested traits compared to the drought-tolerant female parents Langhuang and Chang7-2 (Fig. 1A). Moreover, wide variations $\left(\sigma_{g}^{2}, \sigma_{e}^{2}\right.$, and $\left.\sigma_{g e}^{2}\right)$ in the two $\mathrm{F}_{2: 3}$ populations were observed for the 10 tested traits $(P<0.01$ or $P<0.05)$ (Supplemental Table 2$)$ and showed a continuous distribution, with absolute values of skewness and kurtosis $<1.0$ (Supplemental Table 1). $H^{2}$ of the 10 tested traits in POP1 and POP2 were predominantly high at 72.79 90.95\% (Supplemental Table 2). The observed extensive phenotypic variations and high heritability suggest that the two $\mathrm{F}_{2: 3}$ populations have diverse genetic backgrounds and are suitable for identifying the genetic factors underlying various morphological traits and yield components in maize under contrasting watering conditions.

\section{Phenotypic relationships among all tested traits}

Analyses showed that the 10 tested traits were significantly correlated to each other, with each trait correlating with seven to nine other traits $(P<0.01$ or $P<0.05)$. Moreover, a total of 42 correlations were identified among the 10 traits, the majority (31) of which were positive associations, except for the 11 correlations involving $\mathrm{PH} / \mathrm{EHPH}, \mathrm{ASI} /$ EHPH, ASI/EW, ASI/GW, ASI/KW, ASI/KR, EW/KR, CW/ $\mathrm{GW}, \mathrm{CW} / \mathrm{KW}, \mathrm{CW} / \mathrm{KR}$, and KW/EL (Fig. 1B). Furthermore, $\mathrm{PH}, \mathrm{EH}, \mathrm{ASI}, \mathrm{CW}, \mathrm{EL}$, and EL of the progeny populations (two $F_{1}$ hybrids and two $F_{2: 3}$ populations) depict a significantly positive correlation to female parents (Langhuang/ Chang7-2) and male parent TS141 $(P<0.01)$. EHPH, EW, $\mathrm{GW}$, and $\mathrm{KW}$, however, were significantly positively correlated to the male parent $(P<0.05$ or $P<0.01)$ and were positively correlated to the female parents $(P>0.05)$ (Supplemental Table 3). This finding shows that the 10 tested traits in the progeny populations are simultaneously controlled by both parents, the degree of effects for these traits in both parents differed, and the degree of the effects of $\mathrm{EHPH}, \mathrm{EW}, \mathrm{GW}$, and $\mathrm{KW}$ in the male parent are evidently larger than the female parent. In addition, dendrogram construction was performed for all 10 tested traits, which demonstrated strong correlations as well as extensive phenotypic variations. This classified the 10 tested traits into two unrooted groups. The biggest cluster included seven tested traits, namely, KW, GW, EW, CW, EL, KR, and ASI, whereas the other three traits $(\mathrm{EH}, \mathrm{PH}$, and $\mathrm{EHPH})$ were clustered together (Fig. 1C). This finding suggests that maize corresponding vegetative organs and reproductive organs are clearly separated during the periods from vegetative growth to reproductive growth, then the shoot apical meristem differentiated into inflorescence meristem. Thereby, 

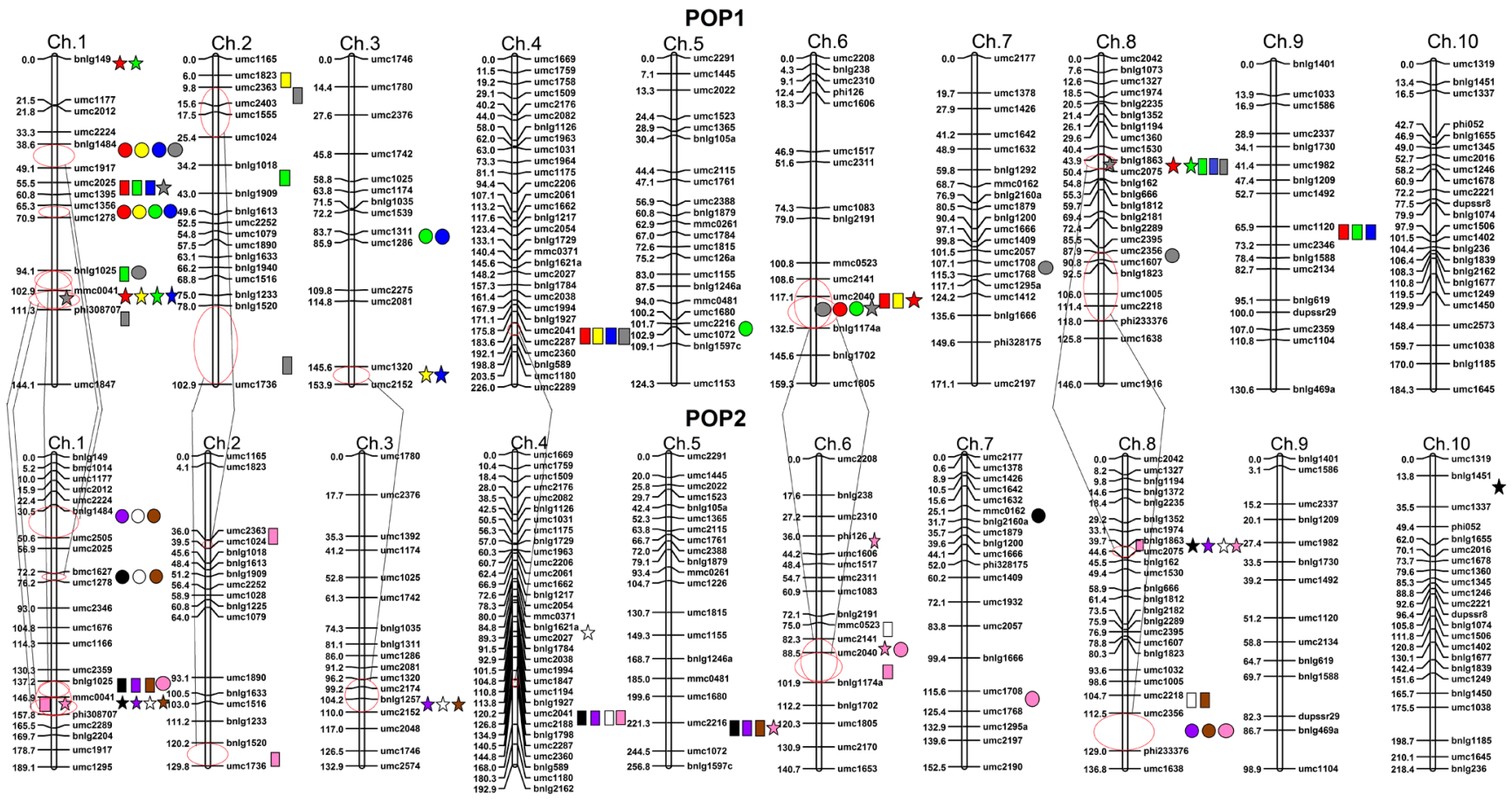

Fig. 2. Genetic linkage map and QTL identification in POP1 and POP2 by single-environment mapping with composite interval mapping (CIM) and joint analysis of all environments with mixed-linear-model-based composite interval mapping (MCIM). Red/yellow/green/blue and black/ purple/white/brown pentagrams, rectangles, and circles represent QTLs for ear height-to-plant height ratio (EHPH), grain weight per ear (GW), and kernel ratio (KR) under well-watered/water-stressed conditions at Wuwei (WW/SW)/Zhangye (WZ/SZ) in POP1 and Gulang (WG/SG)/ Jingtai (WJ/SJ) in POP2 with CIM, respectively. Gray and pink pentagrams, rectangles, and circles represent QTLs for EHPH, GW, and KR in POP1 and POP2 with MCIM, respectively. The black line and red empty oval were combined to a bridge to identify stable QTLs (sQTLs) from both $\mathrm{F}_{2: 3}$ populations.

$\mathrm{PH}, \mathrm{EH}$, and EHPH stop growing, and the inflorescences (tassel and ear) start growing in the following stages.

\section{Identification of QTLs for EHPH, GW, and KR}

To investigate the genetic control underlying EHPH, $\mathrm{GW}$, and KW improvement using single-environment mapping with CIM, we identified 32 significant $(P<0.05)$ QTLs (10 for EHPH, 13 for GW, and 9 for KR) across the two $\mathrm{F}_{2: 3}$ populations (POP1 and POP2) under eight watering conditions (WW, SW, WZ, SZ, WG, SG, WJ, and SJ). The phenotypic variance explained by individual QTLs ranged from $4.07 \%$ to $13.64 \%$ within each watering condition. In addition, 21 QTLs $(65.6 \%)$ were identified under waterstressed conditions (Fig. 2, Supplemental Fig. 2, Supplemental Table 4). For these identified QTLs, approximately 26.1, 65.2, and 8.7\% of the QTLs for EHPH showed additive (A), partial dominance (PD), and dominance (D) effects, respectively. Approximately 25.0, 35.7, 17.9, and $21.4 \%$ of the QTLs for GW showed A, PD, D, and over-dominance (OD) effects, respectively. Approximately $66.7,19.0$, and $14.3 \%$ of the QTLs for KR showed A, PD, and D effects, respectively (Fig. 3). Moreover, all alleles for an increase in EHPH and GW and $42.9 \%$ alleles for a decrease in KR were contributed by male parent TS141 (Supplemental Table 4).

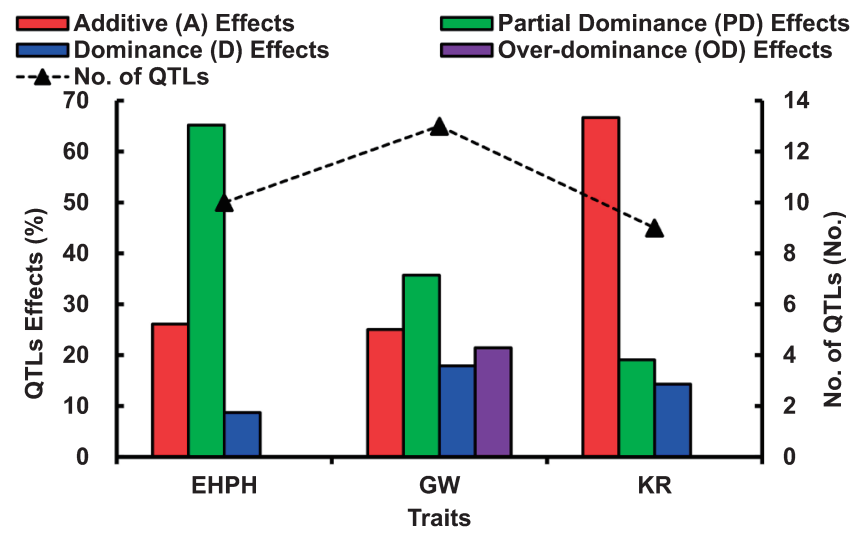

Fig. 3. Summary of identified QTLs for ear height-to-plant height ratio $(\mathrm{EHPH})$, grain weight per ear $(\mathrm{GW})$, and kernel ratio $(\mathrm{KR})$ in the two $\mathrm{F}_{2: 3}$ populations (POP1 and POP2) by single-environment mapping with composite interval mapping (CIM), including number of QTLs and QTLs effects (A, additive effect; PD, partial dominance effect; D, dominance effect; OD, over-dominance effect), respectively.

\section{Identification of joint QTLs and QTL $\times$ E interactions}

Using joint analysis of all watering environments with MCIM, we identified 29 significant $(P<0.05)$ QTLs $(9$ for $\mathrm{EHPH}, 11$ for $\mathrm{GW}$, and 9 for $\mathrm{KR}$ ) in the two $\mathrm{F}_{2: 3}$ populations (POP1 and POP2). Eleven of the 29 QTLs were consistent with those of EHPH, GW, and KR through single watering 
Table 1. QTLs for EHPH, GW, and KR were identified in POP1 and POP2 by joint analysis among all environments with MCIM, respectively

\begin{tabular}{|c|c|c|c|c|c|c|c|c|c|c|c|c|}
\hline \multirow{2}{*}{ Trait } & \multirow{2}{*}{ QTL } & \multirow{2}{*}{ Chr. } & \multicolumn{3}{|c|}{ QTL position } & \multirow{2}{*}{$\mathrm{A}^{b}$} & \multirow{2}{*}{$\mathrm{AE} 1^{c}$} & \multirow{2}{*}{$\mathrm{AE} 2^{c}$} & \multirow{2}{*}{$\mathrm{AE}^{c}$} & \multirow{2}{*}{$\mathrm{AE} 4^{c}$} & \multirow{2}{*}{$\begin{array}{c}h^{2}(A) \\
(\%)^{d}\end{array}$} & \multirow{2}{*}{$\begin{array}{c}h^{2}(A E) \\
(\%)^{e}\end{array}$} \\
\hline & & & $\mathrm{cM}$ & $\mathrm{Mb}^{a}$ & Marker interval & & & & & & & \\
\hline & & & & & POP1 (No. of POP1 & $=202)$ & & & & & & \\
\hline \multirow[t]{4}{*}{ EHPH } & qEHPH-J1-1 & 1 & 56.4 & 0.06 & umc2025-umc1395 & -1.51 & -0.38 & & -0.40 & -0.27 & 9.14 & 4.30 \\
\hline & qEHPH-Ch.1-2 & 1 & 110.7 & 0.40 & mmc0041-phi308707 & -1.07 & & & & & 7.52 & \\
\hline & qEHPH-Ch.6-1 & 6 & 119.8 & 0.03 & umc2040-bnlg1174a & -0.94 & & & & & 7.36 & \\
\hline & qEHPH-Ch.8-1 & 8 & 45.5 & 0.01 & bnlg1863-umc2075 & -1.15 & & & & & 7.89 & \\
\hline \multirow[t]{5}{*}{ GW } & qGW-J1-1 & 1 & 114.2 & 5.03 & phi308707-umc1847 & -1.20 & -0.64 & -0.37 & 0.45 & & 8.14 & 5.01 \\
\hline & qGW-J2-1 & 2 & 10.5 & 0.39 & umc2363-umc 2403 & -0.53 & & & & & 4.85 & \\
\hline & qGW-J2-2 & 2 & 101.8 & 0.24 & bnlg1520-umc1736 & -1.55 & & -0.86 & -0.40 & -0.71 & 9.31 & 6.86 \\
\hline & qGW-Ch.4-1 & 4 & 181.9 & 1.32 & umc2041-umc2287 & -1.31 & & & & & 8.32 & \\
\hline & qGW-Ch.8-1 & 8 & 44.2 & 0.01 & bnlg1863-umc 2075 & -0.35 & & -0.26 & & -0.17 & 2.79 & 1.24 \\
\hline \multirow[t]{6}{*}{$\mathrm{KR}$} & qKR-Ch.1-1 & 1 & 40.7 & 1.56 & bnlg1484-umc1917 & 1.11 & & & & & 11.69 & \\
\hline & qKR-J1-1 & 1 & 95.4 & 2.30 & bnlg1025-mmc0041 & 0.60 & & & 0.37 & & 5.30 & 2.73 \\
\hline & qKR-Ch.6-1 & 6 & 119.8 & 0.03 & umc2040-bnlg1174a & 0.47 & & & & & 3.51 & \\
\hline & qKR-Ch.7-1 & 7 & 110.5 & 1.01 & umc1708-umc1768 & 0.65 & & & & & 5.37 & \\
\hline & qKR-J8-1 & 8 & 88.3 & 0.54 & umc2356-umc1607 & 0.84 & 0.26 & 0.43 & & -0.31 & 8.14 & 2.20 \\
\hline & $a$ FHPH_Ch 1-1 & 1 & 1532 & 040 & POP2 (No. of POP2 & $=218)$ & & & & & 1019 & \\
\hline \multirow{4}{*}{ ЕHРH } & qEHPH-J5-1 & 5 & 239.4 & 0.84 & umc2216-umc1072 & -0.44 & & & & & 4.83 & \\
\hline & qEHPH-J6-1 & 6 & 40.5 & 0.48 & phi126-umc1606 & -0.63 & & -0.22 & & -0.42 & 5.48 & 4.15 \\
\hline & qEHPH-J6-2 & 6 & 87.8 & 0.17 & umc2141-umc2040 & -0.56 & -0.39 & -0.35 & & & 5.12 & 4.29 \\
\hline & qEHPH-Ch.8-1 & 8 & 40.3 & 0.01 & bnlg1863-umc 2075 & -0.71 & & -0.38 & & -0.35 & 5.53 & 4.26 \\
\hline \multirow[t]{6}{*}{ GW } & qGW-J1-1 & 1 & 154.9 & 0.04 & mmc0041-phi308707 & -2.03 & -0.84 & -0.66 & -0.41 & -0.68 & 11.07 & 5.50 \\
\hline & qGW-J2-1 & 2 & 38.6 & 2.01 & umc2363-umc1024 & -0.88 & & & & & 7.31 & \\
\hline & qGW-J2-2 & 2 & 127.1 & 0.24 & bnlg1520-umc1736 & -1.63 & -0.89 & -0.41 & -0.64 & -0.50 & 9.95 & 4.19 \\
\hline & qGW-Ch.4-1 & 4 & 120.3 & 0.66 & umc2041-umc2188 & -1.77 & & & & & 10.14 & \\
\hline & qGW-J6-1 & 6 & 97.4 & 0.03 & umc2040-bnlg1174a & -1.05 & & & & & 8.96 & \\
\hline & qGW-J8-1 & 8 & 40.7 & 0.01 & bnlg1863-umc2075 & -0.84 & -0.36 & -0.52 & & & 7.20 & 4.47 \\
\hline \multirow[t]{4}{*}{$\mathrm{KR}$} & qKR-J1-1 & 1 & 139.3 & 2.30 & bnlg1025-mmc0041 & 0.51 & 0.26 & 0.39 & & & 4.15 & 2.93 \\
\hline & qKR-J6-1 & 6 & 88.1 & 0.17 & umc2141-umc2040 & 0.58 & & & & & 4.20 & \\
\hline & qKR-J7-1 & 7 & 118.0 & 1.01 & umc1708-umc1768 & 0.73 & & & & & 6.12 & \\
\hline & qKR-J8-1 & 8 & 114.9 & 1.96 & umc2356-phi233376 & 0.62 & 0.37 & 0.18 & & & 5.04 & 2.11 \\
\hline
\end{tabular}

EHPH, ear height-to-plant height ratio; GW, grain weight per ear; KR, kernel ratio.

MCIM, mixed-linear-model based composite interval mapping.

${ }^{a} \mathrm{Mb}$ denotes the estimated physical distance of marker interval.

${ }^{b}$ A denotes the additive effect of corresponding QTL.

${ }^{c}$ AE1-4 denote the additive by designated environment (1, well-watered condition at Wuwei in 2014 (WW)/Gulang in 2015 (WG); 2, waterstressed condition at Wuwei in 2014 (SW)/Gulang in 2015 (SG); 3, well-watered condition at Zhangye in 2014 (WZ)/Jingtai in 2015 (WJ); 4, water-stressed condition at Zhangye in 2014 (SZ)/Jingtai in 2015 (SJ) in the POP1/POP2) interaction effects.

${ }^{d} h^{2}(A)$ denotes phenotypic variance explained by the QTL across all environments.

${ }^{e} h^{2}(A E)$ denotes phenotypic variance explained by the additive by designated environment interaction effects.

environment mapping with CIM. The additive effect of each significant QTL ranged from -2.03 to 1.11 , and the phenotypic variance contributed by $\left[h^{2}(A)\right]$ varied between $2.79 \%$ and 11.69\% (Table 1, Fig. 2). Moreover, 14 significant $(P<0.05)$ QTLs (4 for EHPH, 6 for $\mathrm{GW}$, and 4 for $\mathrm{KR}$ ) were involved in QTL $\times$ E interactions in the two $\mathrm{F}_{2: 3}$ populations, and accounted for $1.24-6.86 \%$ of the phenotypic variance explained by $\left[h^{2}(A E)\right]$ (Table 1 ). These QTLs may thus impart stronger effects on EHPH, GW, and KR. For the two $F_{2: 3}$ populations, bin 1.08-1.10 (phi308707-umc1847/ mmc0041-phi308707), bin 2.09 (bnlg1520-umc1736), and bin 8.03 (bnlg1863-umc2075) exhibited three stable QTL $\times$ E interactions for $\mathrm{GW}$, and bin 1.07-1.08 (bnlg1025mmc0041) and bin 8.06 (umc2356-umc1607/umc2356phi233376) exhibited two stable QTL $\times \mathrm{E}$ interactions for KR (Table 1). These findings imply that the five bin intervals may be mediated by environmental factors.

Further analysis showed that 20 sQTLs were simultane- ously identified by single-environment mapping with CIM and joint analysis through MCIM in two $\mathrm{F}_{2: 3}$ populations and were located on chromosomes 1, 2, 3, 4, 6, 8, and 9 . Each sQTL explained 4.15 5.30\% (sQTL17) to 8.81 $13.64 \%$ (sQTL15) of the observed phenotypic variance (Table 2). Approximately 15 (sQTL2, 3, and 5 for EHPH; sQTL6, 7, 8, 10, 11, 12, 13, and 14 for GW; sQTL15, 16, 18 , and 20 for KR) of the 20 sQTLs were identified under water-stressed conditions. This finding indicates that these intervals may have several stable alleles that are involved in plant development and grain yield formation under stress environments. In addition, bin 1.08-1.10 (mmc0041phi308707)/bin 1.07-1.10 (bnlg1025-phi308707) exhibited one common SQTL that imparted a pleiotropic effect on EHPH and GW; bin 6.05 (umc2141-bnlg1174a)/bin 6.05 (umc2040-bnlg1174a) included one common sQTL that imparted a pleiotropic effect on EHPH, GW, and KR; bin 8.03 (bnlg1025-umc2075) mapped one common sQTL that 
Table 2. Summary of stable QTLs (sQTLs) for all investigated traits in POP1 and POP2

\begin{tabular}{|c|c|c|c|c|c|c|c|}
\hline Trait & sQTL & Population & Environment & QTL & Bin & Marker interval & $R^{2}(\%)^{a}$ \\
\hline \multirow[t]{5}{*}{ EHPH } & sQTL1 & POP1 & WW, WZ & qEHPH-Ch.1-1 & $1.00-1.01$ & bnlg149-umc1177 & $6.94 \sim 9.09$ \\
\hline & sQTL2 & POP1, POP2 & $\begin{array}{l}\text { WW, SW, WZ, SZ, WG, SG, WJ, } \\
\text { SJ, Joint }\end{array}$ & qEHPH-Ch.1-1, qEHPH-Ch.1-1 & $1.08-1.10$ & mmc0041-phi308707 & $4.82 \sim 10.38$ \\
\hline & sQTL3 & POP1, POP2 & SW, SZ, SG, WJ, SJ & qEHPH-Ch.3-1 & $3.08-3.09$ & umc1320-umc2152 & $4.14 \sim 11.23$ \\
\hline & sQTL4 & POP1, POP2 & WW, Joint & qEHPH-Ch.6-1, qEHPH-J6-2 & 6.05 & umc2141-bnlg1174a & $5.12 \sim 7.86$ \\
\hline & sQTL5 & POP1, POP2 & WW, WZ, WG, SG, WJ, Joint & qEHPH-Ch.8-1 & 8.03 & bnlg1863-umc2075 & $5.48 \sim 9.89$ \\
\hline \multirow[t]{9}{*}{ GW } & sQTL6 & POP1 & WW, WZ, SZ & qGW-Ch.1-1 & 1.05 & umc2025-umc1395 & $7.25 \sim 9.96$ \\
\hline & sQTL7 & POP1, POP2 & WZ, WG, SG, SJ, Joint & $\begin{array}{l}\text { qGW-Ch.1-2, qGW-Ch.1-1, } \\
\text { qGW-J1-1 }\end{array}$ & $1.07-1.10$ & bnlg1025-phi308707 & $5.99 \sim 11.22$ \\
\hline & sQTL8 & POP1, POP2 & SW, Joint & qGW-Ch.2-1, qGW-J2-1 & $2.02-2.04$ & umc23 & 4.85 \\
\hline & sQTL9 & POP1, POP2 & Joint & qGW-J2-2, qGW-J2-1 & 2.09 & bnlg1520-umc1736 & $9.31 \sim 9.95$ \\
\hline & sQTL10 & POP1, POP2 & WW, SW, SZ, WG, SG, WJ, Joint & qGW-Ch.4-1 & $4.08-4.09$ & umc2041-umc2287 & $4.07 \sim 12.90$ \\
\hline & sQTL11 & POP1, POP2 & WW, SW, WJ, Joint & qGW-Ch.6-1, qGW-J6-1 & 6.05 & umc2040-bnlg1174a & $6.96 \sim 9.23$ \\
\hline & sQTL12 & POP1, POP2 & WZ, SZ, Joint & qGW-Ch.8-1, qGW-J8-1 & 8.03 & bnlg1863-umc 2075 & $2.79 \sim 12.42$ \\
\hline & sQTL13 & POP2 & WJ, SJ & qGW-Ch. $8-1$ & 8.06 & umc2218-umc 2356 & $9.79 \sim 10.55$ \\
\hline & sQTL14 & POP1 & WW, WZ, SZ & qGW-Ch.9-1 & $9.04-9.06$ & umc1120-umc2134 & $6.34 \sim 11.02$ \\
\hline \multirow[t]{6}{*}{$\mathrm{KR}$} & sQTL15 & POP1, POP2 & WW, SW, SZ, SG, WJ, SJ & qKR-Ch.1-1 & 1.03 & bnlg1484-umc2505 & $8.81 \sim 13.64$ \\
\hline & sQTL16 & POP1, POP2 & WW, SW, WZ, SZ, WG, SG, SJ & qKR-Ch.1-2 & 1.07 & umc1356-umc1278 & $4.10 \sim 12.19$ \\
\hline & sQTL17 & POP1, POP2 & Joint & qKR-J1-1 & $1.07-1.08$ & bnlg1025-mmc0041 & $4.15 \sim 5.30$ \\
\hline & sQTL18 & POP1 & WZ, SZ & qKR-Ch.3-1 & $3.06-3.07$ & umc1311-umc1286 & $5.02 \sim 5.33$ \\
\hline & sQTL19 & POP1, POP2 & WW, WZ, Joint & qKR-Ch.6-1, qKR-J6-1 & 6.05 & umc2141-bnlg1174a & $3.51 \sim 11.06$ \\
\hline & sQTL20 & POP1, POP2 & SG, SJ, Joint & qKR-J8-1, qKR-Ch.8-1 & 8.06 & umc2356-phi233376 & $5.04 \sim 10.27$ \\
\hline
\end{tabular}

EHPH, ear height-to-plant height ratio; GW, grain weight per ear; KR, kernel ratio.

WW, well-watered condition at Wuwei in 2014; SW, water-stressed condition at Wuwei in 2014; WZ, well-watered condition at Zhangye in 2014; SZ, water-stressed condition at Zhangye in 2014; WG, well-watered condition at Gulang in 2015; SG, water-stressed condition at Gulang in 2015; WJ, well-watered condition at Jingtai in 2015; SJ, water-stressed condition at Jingtai in 2015; Joint, the joint QTLs were identified across all water environments by the QTL Network version 2.

${ }^{a} R^{2}$ represents the phenotypic variance explained by the QTL.

placed a pleiotropic effect on EHPH and GW; and bin 8.06 (umc2218-umc2356)/bin 8.06 (umc2356-phi233376) identified one common SQTL that had a pleiotropic effect on GW and KR, respectively (Table 2, Fig. 2). These findings indicate that these bin intervals with QTLs control two or more tightly linked traits.

\section{Epistatic interactions among identified QTLS}

For the two $\mathrm{F}_{2: 3}$ populations (POP1 and POP2), 2/1 pair of significant $(P<0.05)$ epistatic interactions for $\mathrm{EHPH}$ were identified with additive by additive (AA)/dominance by dominance (DD) effects under multiple environments, $2 / 1$ pairs of significant $(P<0.05)$ epistatic interactions for $\mathrm{GW}$ were detected with AA/additive by dominance (AD) effects across multiple environments, and 5 pairs of significant $(P<0.05)$ epistatic interactions for KR were mapped with AA effects among multiple environments, respectively. Each epistatic effect explained $2.24 \sim 6.01 \%$ of the observed phenotypic variance contributed by $\left[h^{2}(A A)\right], 3.61 \sim 4.93 \%$ of the phenotypic variance contributed by $\left[h^{2}(A D)\right]$, and $4.08 \sim 4.21 \%$ of the phenotypic variance contributed by $\left[h^{2}(D D)\right]$, respectively (Table 3, Fig. 4). These findings indicate that the main effects of significant QTLs may be stronger on EHPH, GW, and KR.

\section{Discussion}

The complex quantitative traits of $\mathrm{PH}, \mathrm{EH}, \mathrm{ASI}, \mathrm{EW}, \mathrm{CW}$,
GW, KW, and EL have been successfully used as selection criteria in breeding drought-tolerant maize. Drought stress can significantly inhibit PH and EH growth, delay ASI, and adversely decrease EW, GW, KW, and EL in maize (Almeida et al. 2014, Bänziger et al. 2000, Ge et al. 2012, Messmer et al. 2009, Zhao et al. 2018b), which agree with our results involving three parents, two $\mathrm{F}_{1}$ hybrids, and two $\mathrm{F}_{2: 3}$ populations under water deprivation (Fig. 1A). Moreover, the suitable $\mathrm{PH}, \mathrm{EH}$, and EHPH can reduce the plant center of gravity to improve lodging, drought tolerance, and yield potential in crops (Begna et al. 2000, He et al. 2016, Khush 1999). Our results indicate that EHPH and KR significantly decreased under drought conditions because the rate of decrease in $\mathrm{EH} / \mathrm{GW}$ (RC, 12.0/9.9\%) was significantly greater than that of $\mathrm{PH} / \mathrm{EW}$ (RC, 8.2/9.6\%) under drought conditions (Fig. 1A). Therefore, these findings imply that maize can significantly regulate the growth and development of the entire plant, tassel, and ear in response to drought.

Genetic insights into morphological traits and yield components are important for breeding high-yielding maize varieties under drought stress. Our results indicate that $\mathrm{PH}$, $\mathrm{EH}, \mathrm{ASI}, \mathrm{CW}, \mathrm{EL}$, and $\mathrm{KR}$ in $\mathrm{F}_{1}$ hybrids are highly correlated to both parents; however, other four traits were highly correlated to the male parent (Supplemental Table 3), which agrees with the results of Si et al. (2009), Wei et al. (2013), and Zhao (2009). Therefore, to cultivate high-yield maize varieties, breeders should carefully select parents with elite traits. 


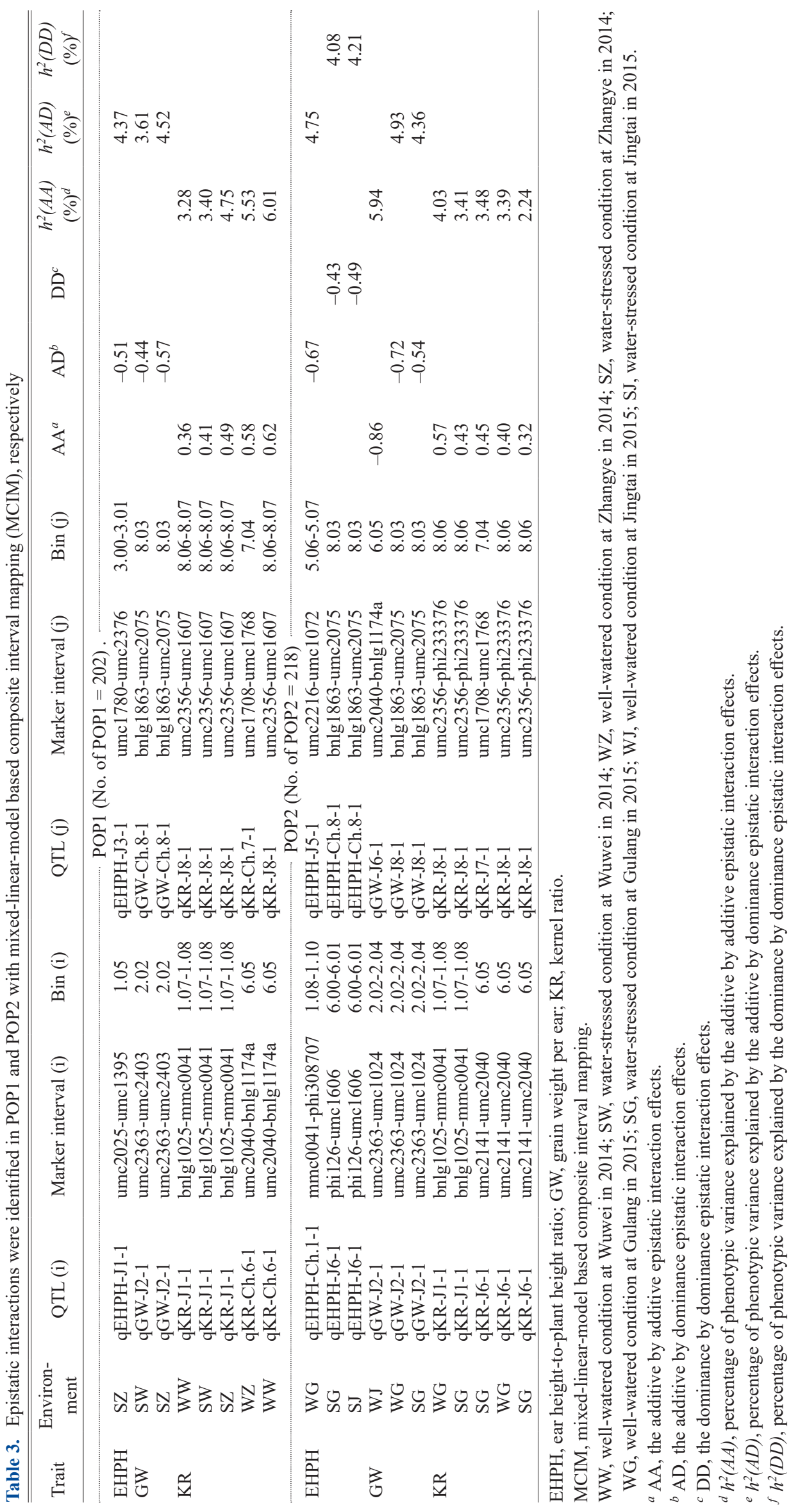



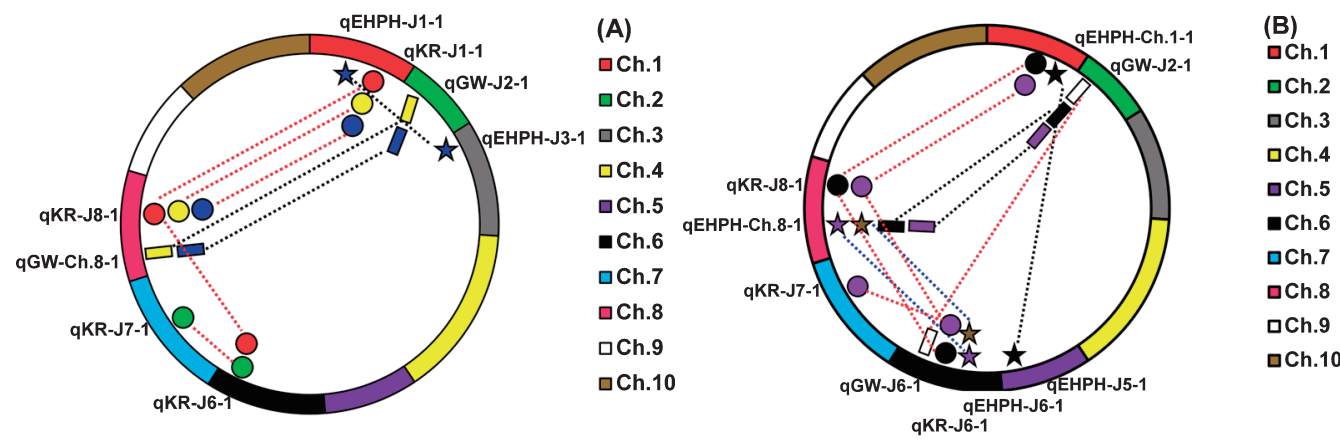

Fig. 4. QTLs with epistatic effects were identified for ear height-to-plant height ratio (EHPH), grain weight per ear (GW), and kernel ratio (KR) in POP1 and POP2 by joint analysis among all environments with mixed-linear-model-based composite interval mapping (MCIM). Red/black/ blue dotted lines represent additive by additive epistatic interaction effects (AA)/additive by dominance epistatic interaction effects (AD)/dominance by dominance epistatic interaction effects (DD). (A) Pentagrams, rectangles, and circles represent corresponding QTLs for EHPH, GW, and KR under well-watered/water-stressed conditions at Wuwei (WW/SW, red/yellow) and well-watered/water-stressed conditions at Zhangye (WZ/ $\mathrm{SZ}$, green/blue) in POP1, respectively. (B) Pentagrams, rectangles, and circles represent corresponding QTLs for EHPH, GW, and KR under well-watered/water-stressed conditions at Gulang (WG/SG, black/purple) and well-watered/water-stressed conditions at Jingtai (WJ/SJ, white/ brown) in POP2, respectively.

Recent genetic improvements in maize grain yield are mostly attributable to increased water stress tolerance of new hybrids, which is achieved by selection for morphological traits and yield components stabilities across target environments, and can be conducted using MAS (Peng et al. 2011). However, genetic investigations on yield components and morphological traits by QTL mapping are often influenced by the environment and genetic background, and even the molecular mechanisms of yield components and morphological traits (especially EHPH, GW, and KR) in maize under different environments remain unclear. Thus, in this study, we detected 32 QTLs for EHPH, GW, and KR based on the two $\mathrm{F}_{2: 3}$ populations under eight watering conditions through single-environment mapping with CIM, which displayed both additive and non-additive effects (Fig. 3). Our findings demonstrate that additive and nonadditive effects, as well as general and specific combining abilities improve EHPH, GW, and KR under both drought and non-drought stress. In addition, 21 of the 32 QTLs were mapped under water-stressed conditions (Supplemental Table 4). It has been suggested that these detected QTLs for EHPH, GW, and KR could be changed under both water regimens, some detected QTLs (e.g. bnlg1621a-umc2027 for $\mathrm{EHPH}$, bnlg1018-bnlg1909 for GW, and mmc0162bnlg2160a for KR) under well-watered conditions were inhibited under water-stressed conditions, whereas some undetected QTLs (e.g., umc1320-umc2152 for EHPH, umc1823-umc2363 for GW, and umc2356-phi233376 for KR) under well-watered conditions were activated under drought stress. Therefore, the QTLs were specifically identified under different watering conditions that directly led to differences in EHPH, GW, and KR. Using joint analysis across environments with MCIM, we detected 29 QTLs for EHPH, GW, and KR across POP1 and POP2, and 11 QTLs were involved in $\mathrm{QTL} \times \mathrm{E}$ interactions, some of these QTL $\times$ E, e.g. qGW-Ch.8-1 in POP1 and qEHPH-Ch.8-1 in
POP2, could also be detected under multiple conditions via single-environment mapping with CIM (Table 1). The observations show that the larger phenotypic variance explained by QTL $\times$ E by joint analysis led to the corresponding QTLs that are easily detected under different environments through single-environment mapping. Further analysis also showed that 20 sQTLs were detected in POP1 and POP2, and 15 of the 20 sQTLs were identified under water-stressed conditions (Table 2). Therefore, these sQTLs can provide reliable information for breeding and future researches on the genetics of EHPH, GW, and KR under different watering environments.

We also noted that the four pleiotropic SQTLs were co-localized with different traits (Table 2, Fig. 2). For example, bin 1.08-1.10 (mmc0041-phi308707, sQTL2)/bin 1.07-1.10 (bnlg1025-phi308707, sQTL17), exhibited one pleiotropic SQTL that simultaneously controlled EHPH and GW. Multiple QTLs for $\mathrm{PH} / \mathrm{PH} / \mathrm{KR}$ were identified in bin 1.07 (umc2151-bnlg1556) (Tang et al. 2007)/bin 1.07 (umc23-umc58) (Lubberstedt et al. 1997, Melchinger et al. 1998)/bin 1.07 (umc1335-umc2236) (Zhang et al. 2013). Using meta-QTL (mQTL) analysis, Dong et al. (2015) reported one $\mathrm{mQTL}$ for EH and top height-to plant height ratio in bin 1.08-1.10 (bnlg1643-umc2189). Li et al. (2011) mapped to one mQTL for EL and kernel number per row in bin 1.07-1.08 (bnlg1556-phi039), and mapped one QTL for ear diameter in bin 1.08-1.09 (bnlg1643-umc2047). Wang et al. (2016) found two mQTLs (bin 1.07 and 1.10) for PH, $\mathrm{EH}$, and yield. Using map-based cloning, Remington et al. (2001) also obtained the indeterminate 1 (idl; bin 1.08) gene that controls $\mathrm{PH}$ growth and development in maize.

In bin 6.05 (umc2141-bnlg1174a/umc2040-bnlg1174a, sQTL4/sQTL19), we identified one pleiotropic sQTL simultaneously affected EHPH, GW, and KR. One sQTL/major QTL for EH was identified in bin 6.05-6.06 (bnlg1732umc2162) (He et al. 2016)/bin 6.05 (bnlg2249-umc1341) 
(Wu et al. 2005). In addition, two mQTLs for yield, ear number per plant, and $\mathrm{GW}$ were validated in bin 6.05 (bnlg1732-bnl8.08c) (Wang et al. 2008, 2016). In bin 6.05 (umc2040-bnlg1174a), Zhao et al. (2017) also found a mQTL affecting ear number per plant and predicted the liguleless 3 (Ig3) gene, which regulates tassel and ear development in maize. In addition, Bauer et al. (2004) elucidated that Ig3 is also a knox class-I homeobox gene that possibly functions in shoot apical meristem maintenance.

In bin 8.03 (bnlg1863-umc2075, sQTL5 and sQTL12), we validated one pleiotropic SQTL that was simultaneously associated with EHPH and GW. Multiple QTLs for kernel depth/PH/KW were identified in bin 8.03-8.05 (umc1302umc1121) (Zhang et al. 2013)/bin 8.03 (umc1741-umc1868) (Wu et al. 2005)/bin 8.03 (bnlg1352-umc1778) (Peng et al. 2011). Two mQTLs for PH, EH, top height, and yield were also detected in bin 8.03 (bnlg1863-phi15) (Dong et al. 2015, Wang et al. 2016). Additionally, Yang et al. (2015) identified one SQTL for ear row number in bin 8.03 (near umc1236), and mapped two mutant genes (vanishing tassel2, vt2 and Barren inflorescence1, bifl) in the interval. Phillips et al. (2011) reported that the vt2 mutant ear typically has barren patches on one or both sides of the ear. Barazesh and McSteen (2008) reported that the mutant gene bif1 plays a role in auxin transport and induces the development of an intermediate (two/more ranked) ear (phyllotaxy).

In bin 8.06 (umc2218-umc2356/umc2356-phi233376, sQTL13/sQTL20), we mapped one pleiotropic sQTL that was simultaneously related to GW and KR. Three QTLs for 10-kernel length/KR/EW were identified in bin 8.06 (umc2175-umc2199) (Peng et al. 2011)/bin 8.06 (umc1960umc1997) (Chang et al. 2017)/bin 8.06 (umc2395mmc0181) (Tan et al. 2011). Moreover, Wang et al. (2016) validated one mQTL for PH, EH, and yield within the same interval and predicted the liguleless 1 (Ig1) and liguleless4 (Ig4) genes in the interval, which have functions similar to that of Ig3 (Bauer et al. 2004, Zhao et al. 2017). Therefore, taken together, the bin 1.07-1.10, 6.05, 8.03, and 8.06 intervals coincided with phenotypic features, thereby supporting pleiotropy of QTLs and playing important roles in conferring growth and yield advantages under contrasting watering conditions.

Epistasis or the interaction effects between a pair of loci may play fundamental roles in broadening our understanding of the genetic mechanism underlying complex quantitative traits (Phillips 2008). In our study, all phenotypic variance was explained by all QTLs for EHPH, GW, and KR, but were always less than the corresponding $H^{2}$, regardless of these traits. This finding implies that the missing proportion of the observed phenotypic variance may be partly explained by epistasis (Zhao et al. 2017). Chang et al. (2017) showed that 15 epistatic interactions for KR are identified based on AA effects. Lan and Chu (2005) reported that 13/14 epistatic interactions for $\mathrm{PH} / \mathrm{EH}$ are identified using $\mathrm{AA}, \mathrm{AD}, \mathrm{DA}$, and dominance by dominance (DD) effects. Lu et al. (2007) found that four epistatic interactions for EL are identified using AA, AD, DA, and DD effects. Yang et al. (2015) reported that two/seven epistatic interactions for ear row number/two-ranked versus many-ranked ear are distinguished by AA effects. Yang et al. (2016) mapped one epistatic interaction for KW that demonstrated an AA effect. Zhao et al. (2017) detected four epistatic interactions for ear number per plant that depicted $\mathrm{AA}$ and $\mathrm{AD}$ effects. In our study, we identified 11 epistatic interactions with $\mathrm{AA}, \mathrm{AD}$, and DD effects among all environments in the two $\mathrm{F}_{2: 3}$ populations (POP1 and POP2). These findings show that AA, $\mathrm{AD}$, and DD may be the main epistatic interaction effects for EHPH, GW, and KR. Notably, five stable epistatic interactions were detected in our study (Table 3, Fig. 4). Namely, one AD epistatic interaction for GW between qGW-J2-1 and qGW-Ch.8-1/qGW-Ch.8-1 was repeatedly identified in POP1/POP2 under four watering conditions; one AA epistatic interaction for KR between qKR-J1-1 and qKR-J8-1 was repeatedly mapped in POP1/POP2 under five watering conditions; one AA epistatic interaction for $\mathrm{KR}$ between qKR-J6-1 and qKR-J7-1 was repeatedly detected in POP1/POP2 under two watering conditions; one AA epistatic interaction for KR between qKR-J6-1 and qKR-J8-1 was repeatedly found in POP1/POP2 under three watering conditions; and one DD epistatic interaction for EHPH between qEHPH-J6-1 and qEHPH-Ch.6-1 was repeatedly identified in POP2 under two watering conditions. These results with the findings of Chang et al. (2017), Lan and Chu (2005), Lu et al. (2007), Sa et al. (2015), Yang et al. (2015), and Yang et al. (2016). Therefore, these five QTL intervals may be considered as epistatic regulators that influence maize plant and ear development under multiple watering conditions.

Here, the three parents, two $F_{1}$ hybrids, and two $F_{2: 3}$ populations were planted under eight different watering environments to analyze the phenotypic variations, and QTLs for corresponding morphological traits and yield components. Then the results show that: (1) the four morphological traits (PH, EH, EHPH, and ASI) and six yield components (EW, CW, GW, KW, EL, and KR) may be used as selection criteria in breeding drought-tolerant maize; (2) The identified QTLs for EHPH, GW, and KR by singleenvironment mapping with CIM and the identified joint QTLs, epistatic interactions, and QTL $\times$ E interactions for the three traits across all environments via MCIM may provide valuable information on the genetic mechanisms of yield components and morphological traits under both drought and non-drought conditions; (3) The identified sQTLs and candidate genes provide reliable information that may be utilized in future studies in identifying quantitative trait genes under contrasting watering conditions. Furthermore, alleles involved in yield components and morphological traits may be used to rapidly generate elite germplasms for MAS, as well as provide a novel strategy for the improvement of maize morphology and yield in arid areas. 


\section{Acknowledgments}

The Scientific Research Start-up Funds for Openly-recuited Doctors, Science and Technology Innovation Funds of Gansu Agricultural University, China (No. GAU-KYQD2018-19), Research Program Sponsored by Gansu Provincial Key Laboratory of Aridland Crop Science, Gansu Agricultural University, China (No. GSCS-2019-8), National Key Research and Development Project of China (No. 2018YFD0100203-4), Developmental Funds of Innovation Capacity in Higher Education of Gansu, China (No. 2019A052), the Fuxi Talent Project of Gansu Agricultural University, China (No. GAUFX-02Y09), and the Major Scientific and Technological Special Project of Gansu, China (No. 17ZD2NA016) supported this study. We thank LetPub for providing linguistic assistance during the preparation of this manuscript.

\section{Literature Cited}

Almeida, G.D., S. Nair, A. Borém, J.Cairns, S. Trachsel, J.M. Ribaut, M. Bänziger, B.M. Prasanna, J. Crossa and R. Babu (2014) Molecular mapping across three populations reveals a QTL hotspot region on chromosome 3 for secondary traits associated with drought tolerance in tropical maize. Mol. Breed. 34: 701-715.

Bänziger, M., G.O. Edmeades, D. Beck and M. Bellon (2000) Breeding for drought and nitrogen stress tolerance in maize: From theory to practice. CIMMYT, Mexico, DF.

Barazesh, S. and P. McSteen (2008) Barren inflorescencel functions in organogenesis during vegetative and inflorescence development in maize. Genetics 179: 389-401.

Bauer, P., M.Lubkowitz, R. Tyers, K. Nemoto, R.B. Meeley, S.A. Goff and M.Freeling (2004) Regulation and a conserved intron sequence of liguleless $3 / 4$ knox class-I homeobox genes in grasses. Planta 219: 359-368.

Begna, S.H., R.I.Hamilton, L.M.Dwyer, D.W. Stewart and D.L. Smith (2000) Variability among maize hybrids differing in canopy architecture for above-ground dry matter and grain yield. Maydica 45: $135-141$

Chang,L.G., K.H.He, T.T.Cui, J.Q.Xue and J.C.Liu (2017) QTL mapping and QTL $\times$ environment interaction analysis of kernel ratio in maize (Zea mays). J. Agric. Biotechnol. 25: 517-525.

Chuck, G.S., P.J. Brown, R. Meeley and S. Hake (2014) Maize SBP-box transcription factors unbranched 2 and unbranched 3 affect yield traits by regulating the rate of lateral primordia initiation. Proc. Natl. Acad. Sci. USA 111: 18775-18780.

Darvasi,A. and M. Soller (1997) A simple method to calculate resolving power and confidence interval of QTL map location. Behav. Genet. 27: 125-132.

Daryanto, S., L. Wang and P.A. Jacinthe (2016) Global synthesis of drought effects on maize and wheat production. PLoS ONE 11: e0156362.

Dong,Y.B., Z.W.Zhang, Q.L.Shi, Q.L.Wang, Q.Zhou, F.Deng, Z.Y.Ma, D.H. Qiao and Y.L.Li (2015) QTL consistency for agronomic traits across three generations and potential applications in popcorn. J. Intergr. Agric. 14: 2547-2557.

Du, Z.H., F.Y.Zhang, J.A. Ping, X. Lv, H.M. Li, Z.X.Tian and T.T. Yang (2011) Correlation analysis of 13 main agronomic characters and drought resistance in corn hybrids. Chinese Agri. Sci. Bull. 27:
$57-63$.

Du, Z.H., F.Y.Zhang, J.A.Ping, X. Lv, H.M.Li, Z.X. Tian and T.T. Yang (2012) Related analysis of main agronomic characters and drought resistance in 31 corn hybrids. Mod. Agric. Sci. Technol. 21: 11-15.

Duvick, D.N. (2005) The contribution of breeding to yield advances in maize (Zea mays L.). Adv. Agron. 86: 83-145.

EI-Soda, M., M.P.Boer, H.Bagheri, C.J.Hanhart, M.Koornneef and M.G.Aarts (2014) Genotype-environment interactions affecting preflowering physiological and morphological traits of Brassica rapa grown in two watering regimes. J. Exp. Bot. 2: 697-708.

Ge, T.A., F.B. Sui, L.C. Bai, C.A. Tong and N.B. Sun (2012) Effects of water stress on growth, biomass partitioning, and water-use efficiency in summer maize (Zea mays L.) throughout the growth cycle. Acta Physiol. Plant. 34: 1043-1053.

He, K.H., L.G.Chang, T.T.Cui, J.Z.Qu, D.W.Guo, S.T.Xu, X.H. Zhang, R.H.Zhang, J.Q. Xue and J.C.Liu (2016) Mapping QTL for plant height and ear height in maize under multi-environments. Scientia Agricultura Sinica 49: 1443-1452.

Khush, G.S. (1999) Green revolution: Preparing for the 21 st century. Genome 42: 646-655.

Knapp, S.J., W.W.Stroup and W.M. Ross (1985) Exact confidence intervals for heritability on a progeny mean basis. Crop Sci. 25: 192-194.

Ku,L., L.Zhang, Z.Tian, S.Guo, H.Su, Z.Ren, Z.Wang, G.Li, X. Wang, Y.Zhu et al. (2015) Dissection of the genetic architecture underlying the plant density response by mapping plant heightrelated traits in mazie (Zea mays L.). Mol. Genet. Genomics 290: $1223-1233$.

Lan, J.H. and D. Chu (2005) Study on the genetic basis of plant height and ear height in maize (Zea mays L.) by QTL dissection. Hereditas 27: 925-934.

Li, J.Z., Z.W.Zhang, Y.L.Li, Q.L.Wang and Y.G.Zhou (2011) QTL consistency and meta-analysis for grain yield components in three generations in maize. Theor. Appl. Genet. 122: 771-782.

Lu,M., F.Zhou, C.X.Xie, M.S.Li, X.H.Li, X.J.Yang, M.Z.Liu and S.H.Zhang (2007) Analysis of epistatic and QTL $\times$ E interaction of ear length in maize. Crops 4: 30-32.

Lu,Y., S.Zhang, T.Shah, C.Xie, Z.Hao, X.Li, M.Farkhari, J.M. Ribaut, M.Cao, T. Rong et al. (2010) Joint linkage-linkage disequilibrium mapping is a powerful approach to detecting quantitative trait loci underlying drought tolerance in maize. Proc. Natl. Acad. Sci. USA 107: 19585-19590.

Lubberstedt,T., A.E. Melchinger, C.C.Schon, H.F.Utz and D. Klein (1997) QTL mapping in testcrosses of European flint lines of maize: I. Comparison of different testers for forage yield traits. Crop Sci. 37: 921-931.

Martin, A., J. Lee, T. Kichey, D. Gerentes, M.Zivy, C. Tatout, F. Dubois, T. Balliau, B. Valot, M. Davanture et al. (2006) Two cytosolic glutamine synthetase isoforms of maize are specifically involved in the control of grain production. Plant Cell 18: 3252-3274.

McCouch, S.R., G.Y.Cho, M. Yano, E. Paul, M. Blinstrub, H. Morishima and T. Kinoshita (1997) Report on QTL nomenclature. Rice Genet. Newsl. 14: 11-13.

Melchinger,A.E., H.F.Utz and C.C.Schon (1998) Quantitative trait locus (QTL) mapping using different testers and independent population samples in maize reveals low power of QTL detection and large bias in estimates of QTL effects. Genetics 149: 383-403.

Meng, Q.L., N.J.Zhao, Y.W.Zhang, Y.Qu, H.F.Zhang and Y.G.Hu (2016) Evaluation of drought resistance on maize inbred lines under special drought conditions. Agri. Res. Arid Areas 34: 106111. 
Messmer, R., Y.Fracheboud, M. Bänziger, M.Vargas, P. Stamp and J.M.Ribaut (2009) Drought stress and tropical maize: QTL-byenvironment interactions and stability of QTLs across environments for yield components and secondary traits. Theor. Appl. Genet. 119: 913-930.

Peng, B., Y.Li, Y.Wang, C.Liu, Z.Liu, W.Tan, Y.Zhang, D.Wang, Y. Shi, B. Sun et al. (2011) QTL analysis for yield components and kernel-related traits in maize across multi-environments. Theor. Appl. Genet. 122: 1305-1320.

Peng, Y.L., X.Q.Zhao, X.W.Ren and J.Y.Li (2014) Genotypic differences in response of physiological characteristics and grain yield of maize inbred lines to drought stress at flowering stage. Agri. Res. Arid Areas 32: 9-14.

Phillips, K.A., A.L.Skirpan, X.Liu, A.Christensen, T.L. Slewinski, C. Hudson, S. Barazesh, J.D. Cohen, S. Malcomber and P. McSteen (2011) vanishing tassel2 encodes a grass-specific tryptophan aminotransferase required for vegetative and reproductive development in maize. Plant Cell 23: 550-566.

Phillips P.C. (2008) Epistasis-the essential role of gene interactions in the structure and evolution of genetic systems. Nat. Rev. Genet. 9: 855-867.

Remington, D.L., J.M. Thornsberry, Y.Matsuoka, L.M.Wilson, S.R. Whitt, J.Doebley, S. Kresovich, M.M. Goodman and E.S. Buckler (2001) Structure of linkage disequilibrium and phenotypic associations in the maize genome. Proc. Natl. Acad. Sci. USA 98: 1147911484.

Ritchie, S.W., J.J.Hanway and G.O. Benson (1997) How a corn plant develops. Spec. Rep. 48. Iowa State Univ. Crop. Ext. Ext. Serv., Ames, IA.

Sa, K.J., J.Y.Park, S.Y.Woo, R.V.Ramekar, C.S.Jang and J.K.Lee (2015) Mapping of QTL traits in corn using a RIL population derived from a cross of dent corn $\times$ waxy corn. Genes Genomics 37: $1-14$.

Semagn, K., Y.Beyene, M.L.Warburton, A. Tarekegne, S. Mugo, B. Meisel, P. Sehabiague and B.M.Prasanna (2013) Meta-analyses of QTL for grain yield and anthesis silking interval in 18 maize populations evaluated under water-stressed and well-watered environments. BMC Genomics 14: 313.

Schmidt, R.J., M.Ketudat, M.J.Aukerman and G.Hoschek (1992) Opaque-2 is a transcriptional activator that recognizes a specific target site in 22-kD zein genes. Plant Cell 4: 689-700.

Si, S.L., X.J.Hao, C. Wei, Y.T. Xia and X.Zhang (2009) The correlation and heterosis of plant-type traits in maize. J. Maize Sci. 17: 51-53.

Stuber, C.W., M.D.Edwards and J.Wendel (1987) Molecular markerfacilitated investigations of quantitative trait loci in maize. II. Factors influencing yield and its component traits. Crop Sci. 27: 639-648.

Su, C., W. Wang, S. Gong, J.Zuo, S. Li and S. Xu (2017) High density linkage map construction and mapping of yield trait QTLs in maize (Zea mays) using the genotyping-by-sequencing (GBS) technology. Front. Plant Sci. 8: 706.

Tan,W.W., Y.Wang, Y.X.Li, Z.Z.Liu, B.Peng, D.Wang, Y.Zhang, B.C. Sun, Y.S. Shi, Y.C. Song et al. (2011) QTL analysis of ear traits in maize across multiple environments. Scientia Agricultura Sinica 44: $233-244$.

Tang, J.H., W.T. Teng, J.B. Yan, X.Q.Ma, Y.J.Meng, J.R. Dai and J.S.Li (2007) Genetic dissection of plant height by molecular markers using a population of recombinant inbred lines in maize. Euphytica 155: 117-124.

Trachsel, S., R. Messmer, P.Stamp and A.Hund (2009) Mapping of
QTLs for leteral and axile root growth of tropical maize. Theor. Appl. Genet. 119: 1413-1424.

Vargas, M., F.A.van Eeuwijk, J.Crossa and J.M.Ribaut (2006) Mapping QTLs and QTL $\times$ environment interaction for CIMMYT maize drought stress program using factorial regression and partial least squares methods. Theor. Appl. Genet. 112: 1009-1023.

Wang, S., C.J.Basten and Z.B.Zeng (2007) Windows QTL Cartographer 2.5. Department of Statistics, North Carolina State University, Raleigh, NC. (http: //statgen.ncsu,edu/qtlcart/WQTLCart.htm)

Wang, X.L., X.H.Li and Z.H. Wang (2008) Construction of integration map and consensus QTL identification for grain yield components in maize. J. Nuclear Agric. Sci. 22: 756-761.

Wang, Y.J., J. Xu, D.X.Deng, H.D. Ding, Y.L.Bian, Z.T. Yin, Y.R.Wu, B.Zhou and Y.Zhao (2016) A comprehensive meta-analysis of plant morphology, yield, stay-green, and virus disease resistance QTL in maize (Zea mays L.). Planta 243: 459-471.

Wei, F., D.F.Hong, Y.Ma, J.F.Ma, X.Y.Wei and X.S.Zhang (2013) Heterosis of plant-type traits in maize. J. Henan Agric. Sci. 42: 1113.

Wu, J.W., C.Liu, Y.S. Shi, Y.C. Song, G.Y.Zhang, Z.Y.Ma, T.Y.Wang and Y.Li (2005) QTL analysis of plant height and ear height in maize under different water regimes. J. Plant Genetic Resources 6: 266-271.

Xing,A.Q., Y.F. Gao, L.F. Ye, W.P.Zhang, L.C.Cai, A.Ching, V.Llaca, B. Johnson, L. Liu, X.H. Yang et al. (2015) A rare SNP mutation in Brachytic2 moderately reduces plant height and increases yield potential in maize. J. Exp. Bot. 66: 3791-3802.

Yang, C., D.G. Tang, L.Zhang, J.Liu and T.Z. Rong (2015) Identification of QTL for ear row number and two-ranked versus manyranked ear in maize across four environments. Euphytica 206: 3347.

Yang, C., L. Zhang, A. Jia and T. Rong (2016) Identification of QTL for maize grain yield and kernel-related traits. J. Genet. 95: 239-247.

Zhang, J., L.X.Ku, Z.P.Han, S.L. Guo, H.J.Liu, Z.Z.Zhang, L.R.Cao, X.J.Cui and Y.H.Chen (2014) The ZmCLA4 gene in the qLA4-1 QTL controls leaf angle in maize (Zea mays L.). J. Exp. Bot. 65: 5063-5076.

Zhang, W.Q., L.X.Ku, J.Zhang, Z.P.Han and Y.H.Chen (2013) QTL analysis of kernel ratio, kernel depth, and 100-kernel weight in maize (Zea mays L.). Acta Agronmica Sinica 39: 455-463.

Zhao, X.Q., Y.L.Peng, J.W.Zhang, P.Fang and B.Y.Wu (2017) Mapping QTLs and meta-QTLs for two inflorescence architecture traits in multiple maize populations under different watering environments. Mol. Breed. 37: 91.

Zhao, X.Q. (2018) Genetic mechanisms study of drought tolerance related to plant architecture in maize (Zea mays L.). Gansu Agricultural University.

Zhao, X.Q., P.Fang, J.W.Zhang and Y.L.Peng (2018a) QTL mapping for six leaf architecture traits under warer-stressed and wellwatered conditions in maize (Zea mays L.). Plant Breed. 137: 60 72.

Zhao, X.Q., Y.L.Peng, J.W.Zhang, P. Fang and B.Y.Wu (2018b) Identification of QTLs and meta-QTLs for seven agronomic traits in multiple maize populations under well-watered and water-stressed conditions. Crop Sci. 58: 507-520.

Zhao, Y.M. (2009) Analysis of genetic effect of kernel rate in maize. Chinese Agri. Sci. Bull. 25: 72-74.

Ziyomo, C. and R.Bernardo (2012) Drought tolerance in maize: indirect selection through secondary traits versus genome wide selection. Crop Sci. 53: 1269-1275. 